\title{
Multicentric glioblastoma in a 4-year-old female patient: A case report
}

\author{
TOSHIYUKI ENOMOTO ${ }^{1,2}$, MIKIKO AOKI ${ }^{1}$, TAKAMICHI KOUZAKI ${ }^{2}$, MASAKI TAKAHARA $^{2}$, \\ HIROSHI ABE ${ }^{2}$, YUKO NOMURA ${ }^{3}$, SHINICHI HIROSE ${ }^{3}$, TOORU INOUE ${ }^{2}$ and KAZUKI NABESHIMA ${ }^{1}$ \\ Departments of ${ }^{1}$ Pathology, ${ }^{2}$ Neurosurgery and ${ }^{3}$ Pediatrics, \\ Fukuoka University School of Medicine, Fukuoka 814-0180, Japan
}

Received March 26, 2020; Accepted January 29, 2021

DOI: $10.3892 / \mathrm{mco} .2021 .2252$

\begin{abstract}
In the USA and Germany, pediatric glioblastoma (pGBM) makes up $<3 \%$ of childhood brain tumors. Occasionally, GBM has multiple contrast lesions and is referred to as multicentric GBM. The current study present a case of a four-year-old female patient presented with headache, vomiting and consciousness disturbance. Radiologically, a neoplastic lesion of the right frontal lobe with hemorrhage, and bilateral thalamus, right temporal and left occipital neoplastic lesions were identified. The right frontal lesion was not continuous to other lesions. It was concluded that the tumor was a multicentric GBM with intra-tumoral hemorrhage. The tumor was pathologically GBM. Following surgery, the patient underwent chemotherapy and radiotherapy, but 11 months after surgery, the patient passed away. This case had features of childhood GBM and multicentric GBM and was difficult to treat.
\end{abstract}

\section{Introduction}

Brain tumor statistics in the USA and Germany has indicated that glioblastoma (GBM) during childhood is relatively rare and accounts for less than $3 \%$ of childhood brain tumors $(1,2)$. Genetic characteristics differ between pediatric GBM (pGBM) and adult GBM (aGBM), and there are differences in five-year survival rates and sensitivity to chemotherapy (2-4). It is possible that pGBM and aGBM, although histologically similar, are different tumors. Also, GBM sometimes has multiple contrast lesions $(5,6)$. In this case, it is classified as multifocal GBM or multicentric GBM depending on whether the contrast area is continuous or independent in high signal areas in T2-weighted images (7). Multiple foci of enhancement embedded within a larger region of T2-weighted signal abnormality is defined as multifocal GBM, and discrete

Correspondence to: Dr Mikiko Aoki, Department of Pathology, Fukuoka University School of Medicine 7-45-1, Nanakuma, Jonan-ku, Fukuoka 814-0180, Japan

E-mail: mikikoss@fukuoka-u.ac.jp

Key words: pediatric, glioblastoma, multicentric enhancing regions without evidence of connecting tumor is defined as multicentric GBM (7). Those with multiple lesions have shorter survival time compared with those with a single lesion (7). Here we report a case of childhood onset and multicentric GBM.

\section{Case report}

The patient was a 4-year-old female, who was born full term and had no problems with growth and development. She had no family history of hereditary disease. One day, she felt a lack of vigor and experienced appetite loss. Two days later, headache and vomiting appeared. After two more days, the vomiting continued and she gradually became drowsy, so she was taken to a nearby doctor. Computed tomography (CT) was performed and revealed an intracranial abnormality, and the patient was transferred to our hospital. On the plain head CT, a $3.5 \mathrm{~cm}$ high-density area was observed and found to be an acute hemorrhagic lesion (Fig. 1A). The bilateral thalamus, right temporal lobe, and left occipital lobe showed low density. On the magnetic resonance image (MRI), the right frontal lobe lesion showed a low signal on a T1-weighted image and a slightly high signal at the edge (Fig. 1B). fluid-attenuated inversion recovery (FLAIR) images revealed regions of high-intensity signal inside the lesion (Fig. 1D). T1-gadolinium (T1-Gd) imaging showed an enhancing effect along the bleeding lesion (Fig. 1C). The bilateral thalamus, right temporal lobe, and left occipital lobe continued with high FLAIR signal (Fig. 1D-F). Within the FLAIR hyperintense region of the left occipital lobe, we found a lesion with a reticulated enhanced effect by T1-Gd imaging (Fig. 1C). Therefore, the lesion was considered to be multiple GBM configured frontal and right temporal-bilateral thalamus-left occipital that caused intratumoral hemorrhage in part, and a craniotomy was performed on the right frontal lobe lesion for the purpose of decompression, diagnosis and preventing rebleeding. Preoperative Karnofsky Performance status (KPS) was 80. The patient was placed in the supine position with her head rotated to the left 40 degrees and supported with a Mayfield headframe. A standard preauricular question mark incision was made. The navigation was used to craniotomy just above the tumor. When the dura mater was incised, we observed a reddish-brown neoplastic lesion. The tumor was hemorrhagic, and a hematoma was found 

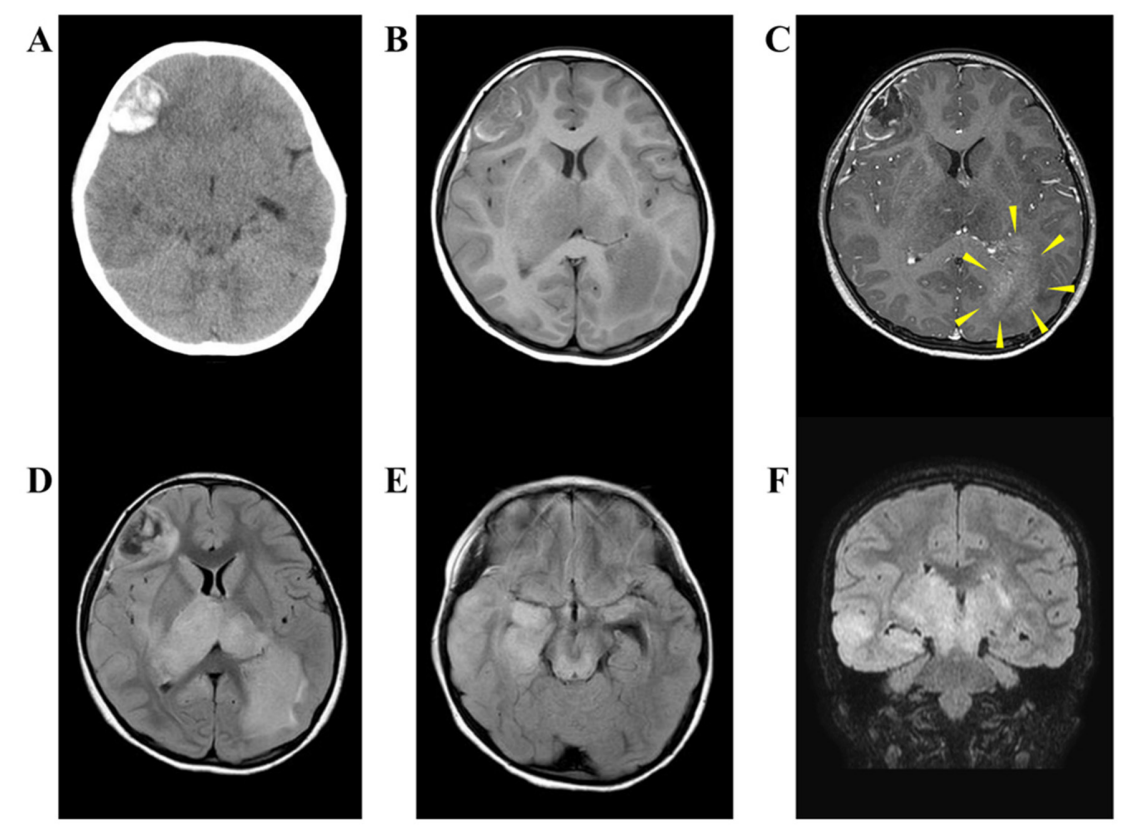

Figure 1. Preoperative CT and MRI. (A) Plain CT shows acute phase hemorrhage of the right frontal lobe. (B) In T1-weighted image of MRI, the frontal lobe lesion shows a low intensity and its margin has a high intensity. Bilateral thalamus and left occipital lobe also show low intensity. (C) Edge of the lesion is enhanced by gadolinium. Reticulated enhanced effect is observed in left occipital lobe (arrow head). (D-F) In the fluid-attenuated inversion recovery MRI, the frontal lesion shows a patchy inside and its margin exhibit a high signal. The bilateral thalamus, right temporal, and left occipital exhibit high intensity and they are continuous. CT, computed tomography; MRI, magnetic resonance image.

A

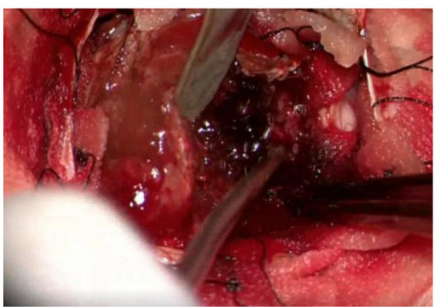

D

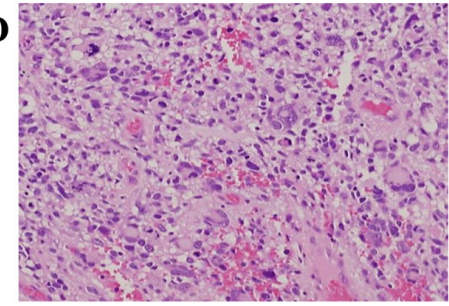

G

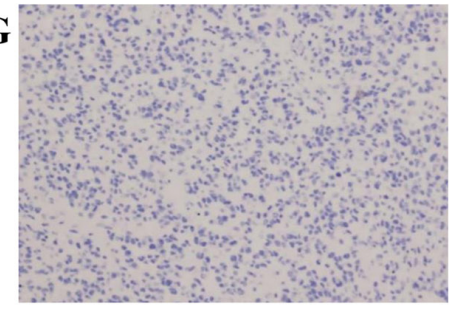

B

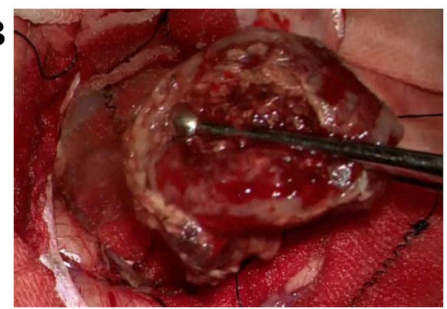

E

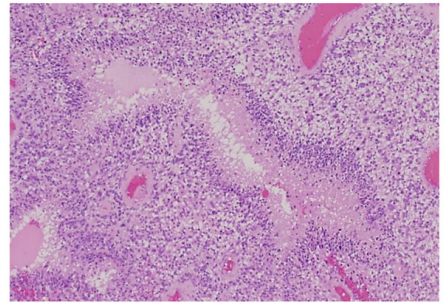

\section{$\mathbf{H}^{5}$}

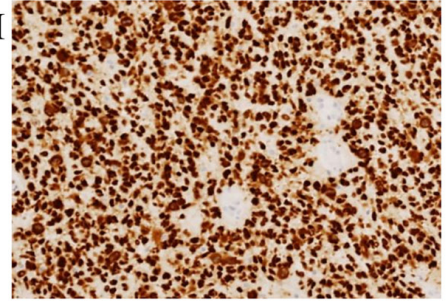

C

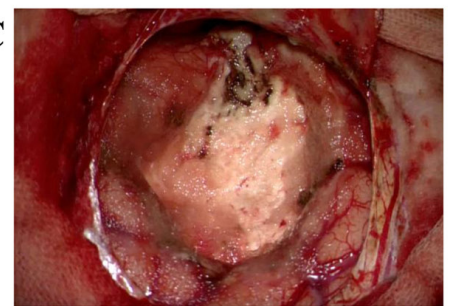

$\mathbf{F}$

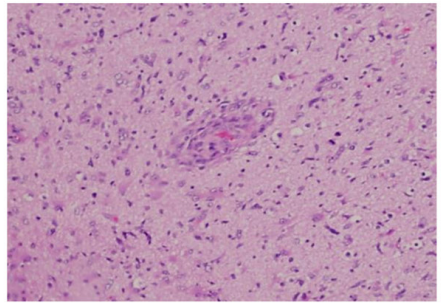

I

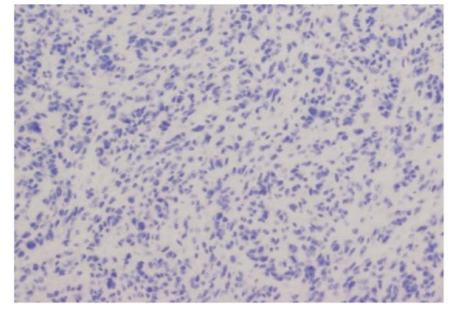

Figure. 2. Operative and histological findings. (A) The tumor was hemorrhagic and intratumoral hematoma was observed. (B and C) The tumor was removed as en bloc. (D) The section shows diffuse proliferation of pleomorphic glial cells. Multinucleated giant cells are visible (hematoxylin and eosin, magnification, $\mathrm{x} 200$ ). (E and F) Palisading necrosis and microvascular proliferations are seen (hematoxylin and eosin, magnification, x40). (G-I) Immunohistochemical analysis. (G) Tumor cells are negative for IDH-1, (H) diffusely positive for p53, (I) and negative for H3 K27M (hematoxylin and eosin, magnification, x100). IDH, isocitrate dehydrogenase.

inside (Fig. 2A). The tumor was clearly demarcated from the surrounding area and was removed as en bloc (Fig. 2B and C).
No surgery was performed on the left occipital lobe lesion. The reason is as follows (1). The lesion responsible for the symptom 

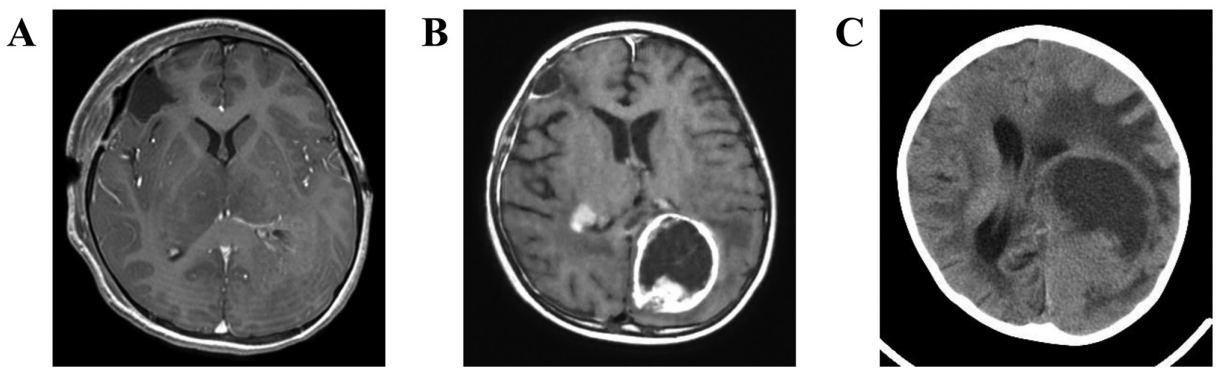

Figure 3. Post-operative scans. (A) MRI the day after surgery. Postoperative enhanced MRI, the lesion in the right frontal lobe was completely removed. (B) A period of 6 months after surgery. The reticulated enhanced area of the left occipital lobe changed to a ring-enhanced lesion, and a new enhanced lesion appeared in right basal ganglia. (C) CT seven months after surgery. The lesion in left occipital robe was enlarged and accompanied by mid-line shift. CT, computed tomography; MRI, magnetic resonance image.

was considered to be a bleeding right frontal lobe lesion (2). The purpose of surgery included decompress, prevention of rebleeding, and pathological diagnosis.

Histopathological and molecular findings. Histopathologically, the tumor samples revealed moderate to high cellularity, and tumor cells had pleomorphic, hyperchromatic nuclei. In addition, we also observed large, highly atypical, multinucleated cells (Fig. 2D). We observed mitotic figures occasionally and palisading necrosis and mild microvascular proliferation (Fig. 2E and F). Immunohistochemically, the tumor cells were negative for IDH-1 (Fig. 2G) and positive for ATRX. P53 was diffusely positive (Fig. 2H) and H3K27M was negative (Fig. 2I). To determine H3F3A, H3K27M, H3G34R, AND H3G34V mutation status we performed QP (QProbe/Qprimer)-Polymerase Chain Reaction using a fully-automated genetic analysis system, the i-densy IS-5320 (Arkray, Inc., Kyoto, Japan). However, no mutations were found. Based on these findings, the tumor was diagnosed as GBM, IDH-wildtype, WHO grade IV. Confirmation of MGMT methylation status of postoperative chemotherapy was performed by immunostaining. The number of cells stained was about $<1 \%$, and Temozolomide was considered to be effective.

Postoperative course. Head MRI performed the day after surgery confirmed the total removal of the right frontal lobe lesion (Fig. 3A). The postoperative KPS was 90 because the symptoms of increased intracranial pressure improved. We performed postoperative treatment with temozolomide (TMZ) and radiation according to the Stupp regimen (8). The radiation dose was reduced to $40 \mathrm{~Gy} / 20 \mathrm{Fr}$ for the child and TMZ was increased from the third-course cycle up to $200 \mathrm{mg} / \mathrm{m}^{2}$. She was given four courses of chemotherapy. At six months after surgery, the FLAIR high signal area and the reticular contrast lesion in the left occipital lobe were both enlarged (Fig. 3B). KPS fell to 40, we stopped chemotherapy and started to palliative care. At seven months after surgery, plane CT revealed further enlarged tumor (Fig. 3C), and she passed away 11 months after the surgery.

\section{Discussion}

This case report is unique in that the patient was a four-year-old suffering from GBM with multiple lesions. pGBM is often associated with neurofibromatosis type 1 ,
Turcot syndrome, and Li-Fraumeni syndrome (9). Although this case did not undergo genetic testing, it was considered to be a relatively rare sporadic case with no family history. GBM has a different prognosis depending on the age of onset, and the five-year survival rate is less than $6 \%$ at 45 years of age or older, but there was a report that the five-year survival rate is $29.8 \%$ in patients younger than five years old (2). In a past study, a subset of pGBM cases that were diagnosed by histopathology more closely resembled pleomorphic xanthoastrocytoma or low-grade glioma in hierarchical clustering of genome-wide DNA methylation (10). Cases in this subset had the BRAF V600E mutation and 9p21 homozygous deletion, or generally balanced genomes in terms of copy number, and long-term survival was observed (10). Therefore, for those cases of pGBM that showed long-term survival, it is possible that detailed examination could reveal biologically distinct characteristics from other, common pGBM cases. When GBM is diagnosed in children, long-term survival is considered difficult if the children do not have special molecular features. Most recently, genomic characterizations have revealed highly prevalent driver mutations in pGBM, including mutations involving histone H3.3, other chromatin remodeling genes, and ACVR1 genes (11). In aGBM, deletion of PTEN and amplification of EGFR are more common but less common in pGBM, and PTEN deletion is considered to be a poor prognostic factor in pGBM (12). IDH1 wildtype and TP53 mutation are also associated with poor prognosis in pGBM $(10,13,14)$. In regards to p53 expression, there are two contradictory reports on whether or not p53 expression is associated with poor prognosis $(13,14)$. For the pGBM case reported in this study, we do not know if there was a deletion of PTEN, but immunohistochemically, IDH1 was negative and p53 was highly expressed. Therefore, it may be possible that the prognosis was genotypically poor.

In addition, this case showed multiple contrast lesions that did not show continuity in high FLAIR signal and presented findings of multicentric GBM. There is no consolidated report on pediatric multicentric GBM, and there is no established treatment. In this case, postoperative radiotherapy and TMZ chemotherapy were performed according to the Stupp regimen. TMZ has an effect of prolonging progression-free survival in pGBM (3). Although it was examined in adult cases, TMZ also prolongs overall survival in multicentric GBM (15), and it was considered to be effective also in this case. Regarding radiation therapy, although there was a report 
that no significant effect was obtained at age five years or less for pGBM (16), radiation therapy was performed at a reduced dose, because only frontal lobe lesions could be removed in our case. The tumor progressed five months after surgery, and this case was considered to be incurable by conventional treatment alone. Recently, an association between pGBM and Biallelic mismatch repair deficiency syndrome and the effectiveness of Nivolumab have been reported (17). For intractable pGBM, further examination of genetic tests and examination of treatment methods are needed in the future.

We experienced a case of pediatric multicentric GBM with intra-tumor hemorrhage. The patient had characteristics of both pediatric and multiple lesion GBM, and the malignancy was difficult to treat. This disease has a poor prognosis, and additional cases need to be accumulated and evaluated to establish an effective treatment regimen.

\section{Acknowledgements}

Not applicable.

\section{Funding}

No funding are received.

\section{Availability of data and materials}

Data sharing is not applicable to this article, as no datasets were generated or analyzed during the present study.

\section{Authors' contributions}

TE drafted the manuscript. MA and KN supervised and reviewed the manuscript. TE, MA and $\mathrm{KN}$ performed the histological examination. TK, MT, HA, TI, YN and SH treated the patient. YN and $\mathrm{SH}$ are treating pediatrician. The authenticity of all the raw data have been assessed by TE, MA and $\mathrm{KN}$ to ensure its legitimacy. All authors read and approved the final version of the manuscript.

\section{Ethics approval and consent to participate}

Not applicable.

\section{Patient consent for publication}

Consent was obtained from family members at the time of surgery for publication of patient data.

\section{Competing interests}

The authors declare that they have no competing interests.

\section{References}

1. Kaatsch P, Rickert CH, Kühl J, Schüz J and Michaelis J: Population-based epidemiologic data on brain tumors in German children. Cancer 92: 3155-3164, 2001
2. Ostrom QT, Gittleman H, Farah P, Ondracek A, Chen Y, Wolinsky Y, Stroup NE, Kruchko C and Barmholtz-Sloan JS: CBTRUS statistical report: Primary brain and central nervous system tumors diagnosed in the United States in 2006-2010. Neuro Oncol 15 (Suppl 2): ii1-ii56, 2013.

3. Walston S, Hamstra DA, Oh K, Woods G, Guiou M, Olshefski RS, Chakravarti A and Williams TM: A multiinstitutional experience in pediatric high-grade glioma. Front Oncol 5: 28, 2015.

4. MacDonald TJ, Aguilera D and Kramm CM: Treatment of high-grade glioma in children and adolescents. Neuro Oncol 13: 1049-1058, 2011.

5. Giannopoulos S and Kyritsis AP: Diagnosis and management of multifocal gliomas. Oncology 79: 306-312, 2010.

6. Hassaneen W, Levine NB, Suki D, Salaskar AL, de Moura Lima A, McCutcheon IE, Prabhu SS, Lang FF, DeMonte F, Rao G, et al: Multiple craniotomies in the management of multifocal and multicentric glioblastoma. Clinical article. J Neurosurg 114: 576-584, 2011.

7. Lasocki A, Gaillard F, Tacey M, Drummond K and Stuckey S: Multifocal and multicentric glioblastoma: Improved characterisation with FLAIR imaging and prognostic implications. J Clin Neurosci 31: 92-98, 2016.

8. Stupp R, Mason WP, van den Bent MJ, Weller M, Fisher B, Taphoorn MJ, Belanger K, Brandes AA, Marosi C, Bogdahn U, et al: Radiotherapy plus concomitant and adjuvant temozolomide for glioblastoma. N Engl J Med 352: 987-996, 2005.

9. Tamber MS and Rutka JT: Pediatric supratentorial high-grade gliomas. Neurosurg Focus 14: e1, 2003.

10. Korshunov A, Ryzhova M, Hovestadt V, Bender S, Strum D, Capper D, Meyer J, Schrimpf D, Kool M, Northcott PA, et al: Integrated analysis of pediatric glioblastoma reveals a subset of biologically favorable tumors with associated molecular prognostic markers. Acta Neuropathol 129: 669-678, 2015.

11. Lam S, Lin Y, Zinn P, Su J and Pan IW: Patient and treatment factors associated with survival among pediatric glioblastoma patients: A surveillance, epidemiology, and end results study. J Clin Neurosci 47: 285-293, 2018.

12. Pollack IF, Hamilton RL, Burger PC, Brat DJ, Rosenblum MK, Murdoch GH, Nikiforova MN, Holmes EJ, Zhou T, Cohen KJ, et al: Akt activation is a common event in pediatric malignant gliomas and a potential adverse prognostic marker: A report from the children's oncology group. J Neurooncol 99: $155-163,2010$.

13. Antonelli M, Buttarelli FR, Arcella A, Nobusawa S, Donofrio V, Oghaki $\mathrm{H}$ and Giangaspero F: Prognostic significance of histological grading, p53 status, YKL-40 expression, and IDH1 mutations in pediatric high-grade gliomas. J Neurooncol 99: 209-215, 2010.

14. Pollack IF, Finkelstein SD, Woods J, Burnham J, Holmes EJ, Hamilton RL, Yates AJ, Boyett JM, Finlay JL and Sposto R; Children's Cancer Group: Expression of p53 and prognosis in children with malignant gliomas. N Engl J Med 346: 420-427, 2002.

15. Syed M, Liermann J, Verma V, Bernhardt D, Bougatf N, Paul A, Rieken S, Debus J and Adeberg S: Survival and recurrence patterns of multifocal glioblastoma after radiation therapy. Cancer Manag Res 10: 4229-4235, 2018.

16. Liu M, Thakkar JP, Garcia CR, Dolecek TA, Wagner LM, Dressler EVM and Villano JL: National cancer database analysis of outcomes in pediatric glioblastoma. Cancer Med 7: 1151-1159, 2018.

17. Bouffet E, Larouche V, Campbell BB, Merico D, de Borja R, Aronson M, Durno C, Krueger J, Cabric V, Ramaswamy V, et al: Immune checkpoint inhibition for hypermutant glioblastoma multiforme resulting from germline biallelic mismatch repair deficiency. J Clin Oncol 34: 2206-2211, 2016. 